\title{
Macht und Verantwortung
}

\author{
Prof. Dr. Rouven Porz
}

Medizinethik und ärztliche Weiterbildung, Insel Gruppe, Inselspital Bern

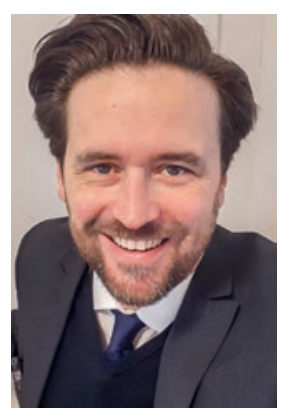

«Mit grosser Macht kommt auch grosse Verantwortung.» Dieser Satz hat mich sehr fasziniert, als ich ihn zum ersten Mal gehört habe. Eigentlich ist die Aussage ja klar: Wenn man als einzelne Person über grosse Macht verfügt, dann muss man auch aufpassen, dass man diese Macht gut einsetzt, am besten verantwortungsvoll und zum grössten Wohl von möglichst vielen. Dennoch, ich hatte zuvor nie über den konkreten Zusammenhang zwischen Macht und Verantwortung nachgedacht und fühlte mich von dem Satz inspiriert. Jetzt stammt dieser aber leider nicht aus einem Lehrbuch für Ethik. Vielmehr handelt es sich um ein Zitat aus einem US-amerikanischen Kino-Blockbuster, konkret, aus einem Spiderman-Film. Der junge Spiderman realisiert in der Szene zum ersten Mal, über welche Superheldenkräfte er verfügt, und sein alter Onkel erklärt ihm mit diesem Satz, dass er seine neuen Kräfte zum Wohle der Menschheit einsetzen soll. Bleibt für mich die Frage: Wieso steht sowas Hilfreiches eigentlich nicht in einem Medizinethik-Lehrbuch?

Verstehen Sie mich nicht falsch - ich meine das nicht ironisch. Jungen Ärztinnen und Ärzten wird weder im Studium noch in ihrer Assistenzarztzeit erklärt, über welche Macht sie verfügen und in welche Machtstrukturen sie im Gesundheitswesen hineinsozialisiert werden. Dabei muss Macht ja gar nichts Schlechtes sein (siehe oben: Spiderman). Diese fehlende Reflexion zum Konzept der Macht ist eigentlich überraschend, wo doch gerade ein Spital ein Ort ist, in dem Machtstrukturen allgegenwärtig sind. Wäre es nicht wichtig, man würde den jungen Ärztinnen und Ärzten genauer erklären, was der Unterschied zwischen struktureller Macht und Gestaltungsmacht ist oder was man sich unter symbolischer Macht vorzustellen hat? Wäre es nicht noch schöner, man würde sie darauf aufmerksam machen, welche Macht sie einmal über Patientinnen und Patienten haben werden und wie sie versuchen könnten, damit verantwortungsvoll umzugehen? Allein der Wissensvorsprung über die Körperfunktionen, über Diagnosen, Therapien und Prävention katapultiert sie in fast jedem zukünftigen Patientengespräch in eine ungefragte - und hoffentlich ungewollte - Machtposition, allein weil sie zum Thema
Krankheit und Gesundheit so viel mehr wissen als die meisten Personen, die vor ihnen sitzen.

Hier ist selbst meine eigene Disziplin, die Medizinethik, ganz schlecht aufgestellt. Auch in der wichtigsten Hintergrundtheorie der klinischen Medizinethik der sogenannten Vier-Prinzipien-Ethik - ist von Macht eigentlich keine Rede. Die Prinzipienethik gruppiert sich um die Anwendung von vier mittleren Prinzipien, die Ärztinnen und Ärzten helfen sollen, einen Reflexionsprozess in moralisch-herausfordernden Situationen zu strukturieren. Die Rede ist hier von den Prinzipien (i) Respekt vor der Patientenautonomie, (ii) Fürsorge, (iii) Nicht-Schaden und (iv) Gerechtigkeit. Diese berufsethischen Prinzipien schnüren zwar ein ethisches Korsett dazu, in welcher Haltung man seinen Patientinnen und Patienten gegenübertreten soll, sie erlauben aber kaum eine Reflexion zur eigenen Rolle als Arzt oder Ärztin im hierarchischen Machtkontext von Kliniken. Damit bleibt die Medizinethik immer sehr in der einzelnen Patientensituation verhaftet und schafft es in ihren Theorien kaum, institutionelle oder organisatorische Themen zu reflektieren. Etwas mehr Reflexion zum Thema Macht findet sich in den sogenannten Care Ethics (manchmal ist hier auch von «Feministischen Zugängen zur Medizinethik» die Rede). Diese Care Ethics basieren nämlich gerade nicht auf einem Menschenbild, in dem nur eine vermeintliche Autonomie der Patientinnen und Patienten im Vordergrund steht. Sie beruhen als Theorie vielmehr auf dem Gedanken, dass es gar keine reine Autonomie gibt und dass unsere gegenseitigen Beziehungen und Abhängigkeiten immer mitbedacht werden müssen. Deshalb haben die Care Ethics auch eine dezidiertere Meinung zur Macht: Macht muss benannt, reflektiert und mitgedacht werden, denn immer, wenn Menschen um einen Tisch zusammensitzen, kommen die einzelnen an den Tisch mit Machtansprüchen und Machtinteressen, sei dies bewusst oder unbewusst. Es bleibt für mich als Schlussfolgerung: Solche Zusammenhänge sollten wir unseren Studierenden und jungen Ärztinnen und Ärzten mehr mit auf den Weg geben. Es kann ja auch nicht sein, dass sie dies in SpidermanFilmen lernen müssen. 